\title{
Complications and their Management in Implantology
}

\author{
Smita Vaidya, Arun Khalikar, Shankar P Dange, Rujit Desai
}

\section{ABSTRACT}

Implant prosthodontics will become straightforward if the fixture position and angulation are ideal. But the prosthetic treatment may be complicated by component and framework breakage, inadequate tissue support, poor implant position and angulation. Complications during maintenance of implant supported prostheses have considerable clinical and laboratory implications. It is important for the practitioner who uses implants to have an understanding of the type and frequency of complications that may arise.

This paper focuses on the common complications in treatments involving the use of implants and also considers the modalities for its management. The practitioner should be fully aware of any possible complications prior to treatment and inform the patient accordingly. In most cases it is possible to avoid complications by careful attention to diagnosis, treatment planning and good surgical and prosthodontic planning.

Keywords: Complications, Management, Implant, Prosthodontics.

How to cite this article: Vaidya S, Khalikar A, Dange SP, Desai R. Complications and their Management in Implantology. Int J Prosthodont Restor Dent 2012;2(4):150-155.

Source of support: Nil

Conflict of interest: None

\section{INTRODUCTION}

The field of implantology is progressing very rapidly with a wide variety of applications in various interdisciplinary branches. These include prosthodontics (for replacing missing tooth and maxillofacial prosthesis), orthodontics (for the purpose of growth studies ${ }^{1}$ and anchorage ${ }^{2}$ ), periodontics (for bone preservation and augmentation ${ }^{3}$ ) and oral surgery. Proper case selection and treatment planning are the keys to success of implants. The focus of implant research is shifting from descriptions of clinical success to the identification of factors associated with failure. ${ }^{4} \mathrm{~A}$ detailed knowledge regarding the complications and failures is a must. Prompt management of these complications holds the key to the success of the implants.

This article reviews the various complications that one faces during implant therapy. It also throws light on the management of the complication. It is necessary to have knowledge regarding the complication as it is the operator to be blamed in case of implant failure.

\section{RISK FACTORS}

Risk factors require an indispensible attention as they are often responsible for implant failures. The risk factors may be stated as:

\section{Mechanical Factors}

- Implant shape, surface

- Titanium implants with different shapes and surface preparations have similar success rates, but that smooth implants, compared to rough implants, appear to be less prone to peri-implantitis. ${ }^{5}$

- Implant length and diameter

- Implants more than $>4 \mathrm{~mm}$ in diameter showed better success rate than those with lesser diameters. ${ }^{5}$

- Shorter implants also showed more failure rates.

\section{Anatomic and Osseous Factors}

- Significant determinants for implant failure were poor bone quality (type 4), a resorbed jaw, and short implant length $(7 \mathrm{~mm}){ }^{5}$

- It has been found that neither jaw site (maxilla vs mandible) nor implant position (anterior vs posterior) had any significant effect on implant survival. ${ }^{5}$

\section{Factors related to Occlusal Loading}

- Parafunctional habits and excessive occlusal loadings are often a risk factor for implant failure.

- The opposing occlusion or dentition may also be a relevant determinant of implant success. Patients with implants opposing unilateral occlusal support showed the highest rate of implant failure (43.8\%). ${ }^{5}$

\section{Systemic Risk Factors}

- The most common systemic risk factors leading to implant failure are smoking, radiation treatment, diabetes (resulting in increased bone loss).

- Other common systemic conditions acting as risk factors for implant failure include chemotherapy, osteoporosis, hormone replacement therapy, scleroderma, Sjogren's syndrome, Parkinson's disease, multiple myeloma and HIV-positive individuals.

\section{Microbial and Host Immune-inflammatory Factors}

- Peri-implantitis, defined as infection and inflammation affecting implant supporting tissues, is leading causes of late implant failures.

- Organisms commonly involved are Porphyromonas gingivalis, Actinobacillus actinomycetemcomitans, Prevotella nigrescens, Staphylococcus aureus, 
Peptostreptococcus micros, Fusobacterium nucleatum, ss vincentii, $F$. nucleatum and ss nucleatum.

\section{COMPLICATIONS}

A complication is defined as a secondary condition that developed during or after implant surgery or prosthesis placement. ${ }^{6}$ It does not indicate that a substandard treatment was provided and also that an implant has failed. Prompt management of the complications is the key to implant success.

In 1999, Charles Goodacre et al, ${ }^{6}$ had classified implant complications as implant loss as related to type of prosthesis and arch, time after placement, implant length, bone quality, surgical complications, marginal bone loss, peri-implant soft tissue complications, mechanical complications, and phonetic and esthetic complications.

In 2008, Kelly Misch ${ }^{7}$ et al, had classified implant as:

- Treatment plan related (wrong angulation, improper implant location, lack of communication),

- Procedure related (lack of primary stability, mechanical complications, mandibular fracture, ingestion/ aspiration),

- Anatomy related (nerve injury, bleeding, cortical plate perforation, sinus perforation, devitalization of adjacent teeth) and others (iatrogenic, human error).

In 2010, Stuart J Froum, ${ }^{8}$ stated implant complications as:

- Associated with systemic disorders and medications

- Associated with implant planning

- Implant fractures

- Implant failures

- Peri-implantitis

- Esthetic complications due to implant malposition

- Related to immediate implant placement into extraction sites

- Related to immediately loaded dental implants

- Complications can also be described as those occurring during first stage surgery, second stage surgery, abutment connection, prosthetic procedure, control after prosthesis placement (Table 1).
A brief review of complications can be stated as:

\section{A. Complication associated with Systemic Disorders and Medications}

The disease often leading to complications are:

i. Myocardial infarction: Alteration in blood and oxygen supply interferes with the process of osseointegration.

ii. Cerebrovascular disease (stroke): No direct role on failure of implants has been documented. ${ }^{8}$

iii. Osteoporosis: The impaired bone metabolism as it occurs in osteoporosis may affect osseointegration of implants.

iv. Paget's disease: Paget's disease has compromised bone density and may be contraindicated for dental implant surgery.

v. Parkinson's disease: Poor motor control, often a cause of improper oral hygiene maintenance.

vi. Diabetes: Studies of implants in the anterior mandible have shown 5-year survival rates of 88 to $94 \%$ in subjects with type II diabetes. ${ }^{8}$

vii. Smoking: Smoking increases the rate of implant complications.

viii. Immunodeficiency: Long-term systemic steroids can induce osteoporosis, which should be considered in the risk-benefit assessment for implant therapy.

ix. Cancer therapy - radiation: When implants are placed following irradiation, the failure rate may be higher.

\section{B. Complication associated with Implant Planning}

a. Lack of proper diagnosis, patient history especially of the systemic conditions is often responsible for the complications of implants.

- Using too few implants can lead to occlusal overload and ultimate failure of the prosthesis. Patient's motivation for cleanliness and implant maintenance also plays a vital role as poor oral hygiene can often result in peri-implantitis.

b. Proper implant planning of treatment is of utmost importance. Improper planning may result in:

Table 1: Implant complications

\begin{tabular}{|c|c|c|}
\hline 1st stage (during surgery) & 2nd stage (abutment connection) & 3rd stage (prosthetic phase) \\
\hline 1. Hemorrhage during drilling & 1. Sensitivity & 1. Loosening of abutment screws \\
\hline 2. Implant mobility after placement & 2. Mobile implant (slight) and painful & 2. Fracture \\
\hline 3. Exposed implant threads & 3. Difficulty in insertion & i. Abutment screw \\
\hline 4. Lingual swelling & 4. Formation of granulation tissue & ii. Veneering material \\
\hline 5. Postoperative pain & around implant & iii. Frame work \\
\hline 6. Lower lip insensitivity & & 3. Bleeding on probing \\
\hline 7. Exposed cover screw after few days & & 4. Implant fracture \\
\hline 8. Abscess around cover screw & & 5. Bone loss around implant \\
\hline
\end{tabular}


- Tooth or root proximity to a planned implant site can cause adjacent tooth devitalization (Fig. 1).

- An adjacent tooth with an undiagnosed periapical lesion could lead to implant failure, when the infection spreads and reaches the implant surface.

- Implant fenestration or dehiscence may occur due to improper placement of implant.

- Another factor of prime importance concerns vital anatomic structures. Structures of importance to note before beginning treatment are the proximity of the inferior alveolar canal, mental foramen, sinus, nasal floor, and incisive canal. Anatomic variations can lead to perforations of the alveolar bone during treatment. This could lead to soft tissue and/or artery damage, with the ensuing complications (Fig. 2).

\section{Implant Fractures}

a. Implant fracture is an infrequent and late biomechanical complication (Fig. 3).

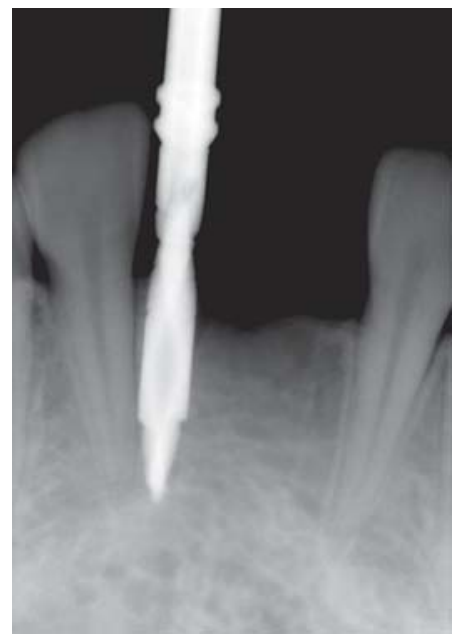

Fig. 1: Improper implant angulation

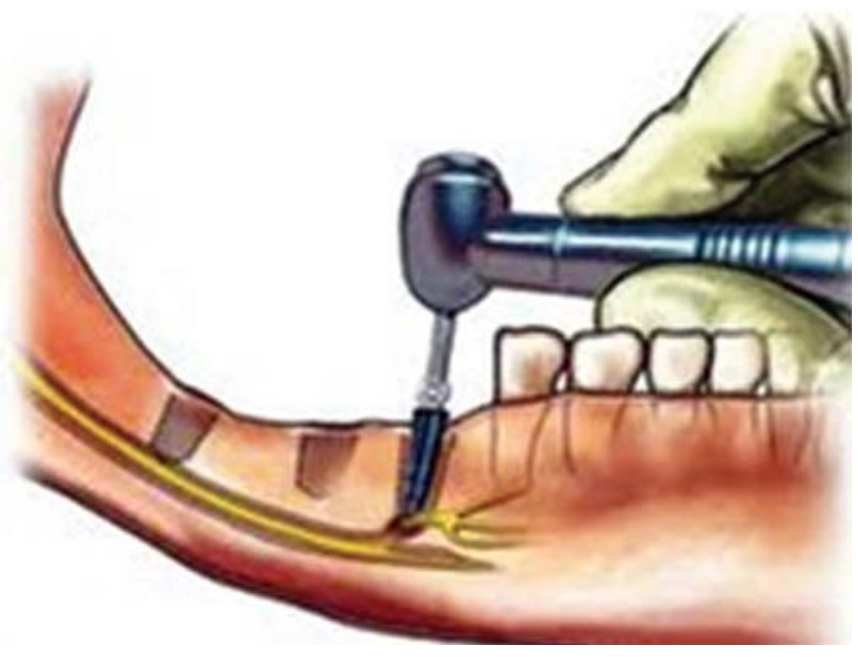

Fig. 2: Perforations of the inferior alveolar canal

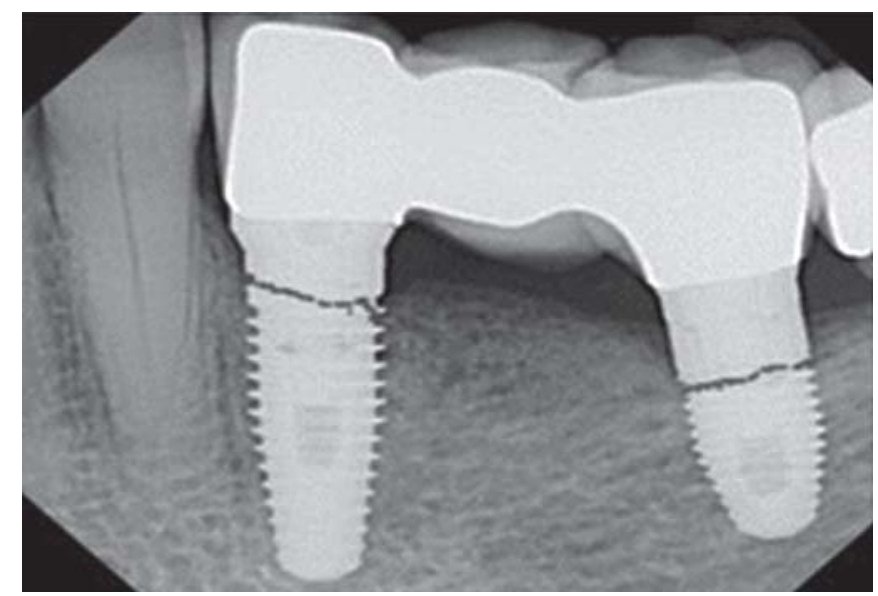

Fig. 3: Implant fracture

b. Incidence: $0.6 \%$ of all implant placements, with a lower incidence in edentulous jaws $(0.2 \%)$ and more frequent occurrence in partially edentulous jaws (1.5\%). ${ }^{8}$

c. Etiology:

i. Bone loss may be a factor that is associated with implant fracture.

ii. Manufacturing defects: Defects in the raw materials and in the manufacturing process are certainly possible when implants are made.

iii. Biomechanics: Excessive occlusal load can lead to implant fracture. Typically, fractured implants are found in the molar areas where this force potential is quite high.

iv. Patient-related habits.

\section{Peri-implantitis}

a. Peri-implant mucositis is a term used to describe reversible inflammatory reactions in the mucosa adjacent to an implant. Peri-implantitis is defined as an inflammatory process that (i) affects the tissues around an osseointegrated implant in function and (ii) results in loss of supporting bone.

b. Diagnostic aspects:
i. Mobility
ii. Bleeding on probing
iii. Increased probing death and loss of attachment iv. Pus formations.

\section{E. Esthetic Complications due to Implant Malposition}

a. Malposition of implant can lead to significant and permanent loss of hard and soft tissue support with extremely adverse esthetic outcomes.

b. Coronoapical malposition (Fig. 4)

- A coronoapical malposition can cause two different complications 
- Superficial implant placement-unesthetic display of metal

- Deep apical malposition of implant can cause recession of the facial mucosa, if the implant only has a thin facial bone wall at implant placement.

c. Orofacial malposition (Fig. 5):

- An orofacial malposition of an implant can also cause two different complications:

- Palatal placement may result in tongue interference

- Facial placement may cause recession of the facial mucosa.

\section{F. Implant Complications related to Immediate Implant Placement into Extraction Sites (Fig. 6)}

Most common complications that occur with immediate implant placement after extraction of the natural tooth include:

- Poor implant positioning

- Membrane exposure during healing

- Inadequate bands of keratinized tissue after healing

- Gingival recession

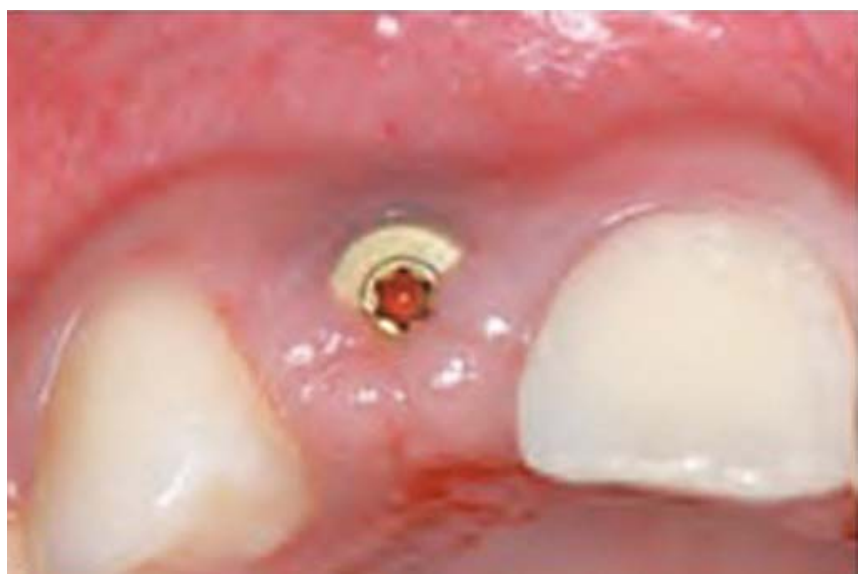

Fig. 4: Coronoapical malposition

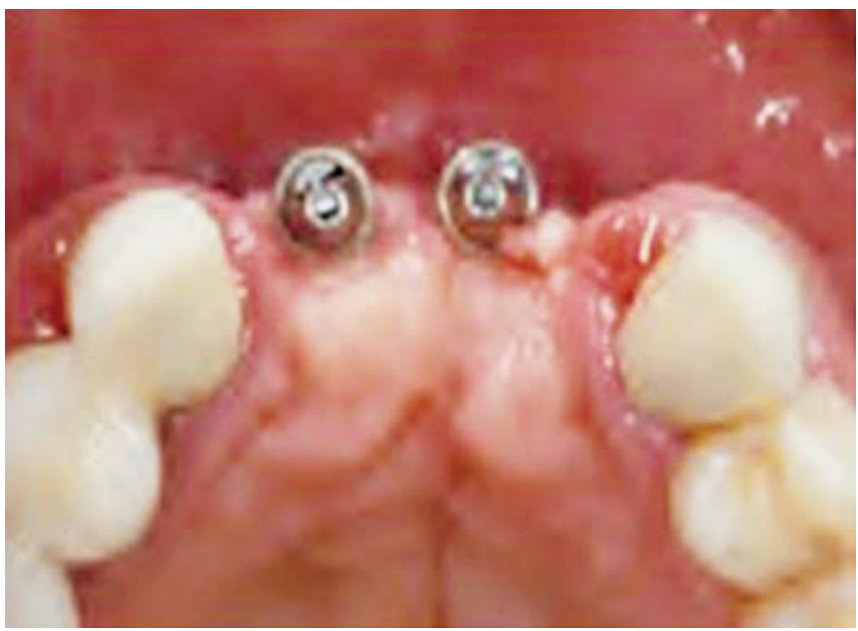

Fig. 5: Facial placement of implant
- Implant failure

- Unacceptable esthetic outcomes.

\section{G. Complications related to Immediately Loaded Dental Implants (Fig. 7)}

Complications addressed in this chapter that are associated with the immediate implant loading protocol include:

- Failure of the implant to osseointegrate

- Surgical complications

- Esthetic complications

- Implant malposition

- Restorative complications

- Complications with guided surgery and prefabricated restorations.

\section{H. Implant Failures}

a. Criteria for implant success were defined by Albrektsson and Zarb ${ }^{9}$ in 1986, followed and modified later by Roos et al. ${ }^{10}$ These criteria for success include:

- No mobility,

- No radiographic evidence of peri-implant translucency,

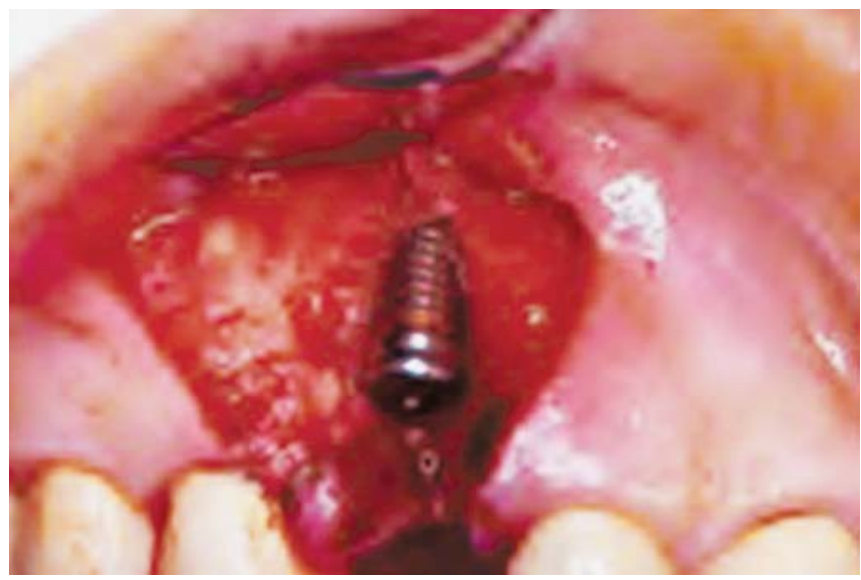

Fig. 6: Loss of labial plate

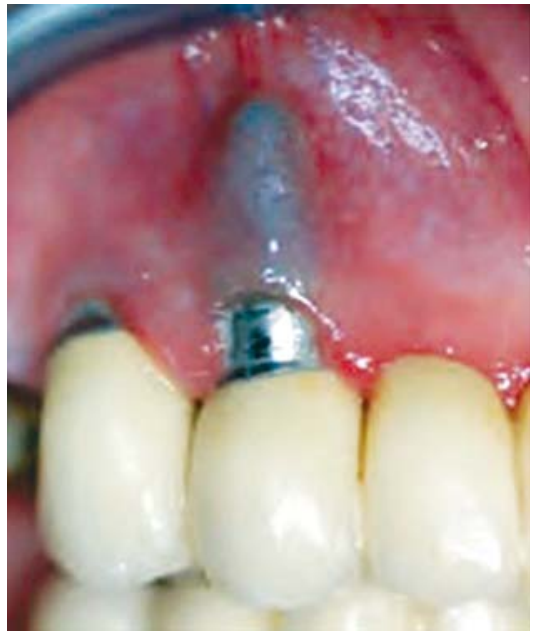

Fig. 7: Extensive facial resorption 
- $\leq 1 \mathrm{~mm}$ bone loss 1 year following implant loading and $\leq 0.2 \mathrm{~mm}$ annually thereafter,

- Absence of pain and pathology around the implant,

- Functional survival for 5 years in $90 \%$ and 10 years in $85 \%$, of cases respectively.

b. Classification of implant failure: There are two commonly used periods to assess an implant failure that relate to the time of occurrence:

- Early failures: Failures before osseointegration, primarily the result of surgical and/or postoperative complications.

- Late failures: Failures after the osseointegration period, usually arising during and after the restorative phase.

c. Incidence: Rosenberg and Torosian ${ }^{11}$ reported an overall failure rate of $7.0 \%$ in a 7.5-year investigation that aimed to identify clinical and/or microbiological differences associated with failure in five different implant systems.

d. Etiology and risk factors: Implant failure can be caused by several factors, including:

- Infection

- Tissue trauma (e.g. overheating of bone, pressure necrosis)

- overload (e.g. transmucosal loading, occlusal trauma)

- Iatrogenic and improper angulations.

\section{MANAGEMENT}

A detailed knowledge of the complication is essential. This will enable its prompt management and thereby ensuring implant success. Management of the stated complication is as follows:

\section{A. Systemic Disorder and Medication-related Complications}

a. Proper case history and systemic evaluation

b. Stop the procedure, in case of complication, during surgery and seek medical help. It is wise to have nitroglycerine, adrenaline and oxygen handy

c. Proper postoperative antibiotic course

d. Regular recalls

e. Abstaining from habits - smoking.

\section{B. Complication associated with Implant Planning}

a. Proper diagnosis, evaluation of patient and treatment planning

b. Necessary investigations should be thoroughly performed c. Patient instructions should be given prior to surgery

d. Manufacturing instructions regarding implant placement should be followed.

\section{Implant Fractures}

Three management options have been described in the event of implant fracture. ${ }^{12}$

i. Complete removal of the fractured implant using explantation trephines.

ii. Removal of the coronal portion of the fractured implant with the purpose of placing a new prosthetic post.

iii. Removal of the coronal portion of the fractured implant, leaving the remaining apical part integrated in the bone.

\section{Peri-implantitis}

a. Cumulative interceptive supportive therapy (CIST): Four-step procedure, along with antibiotics.

1. Mechanical debridement

2. Antiseptic treatment

3. Antibiotic treatment

4. Regenerative or resective therapy.

b. Removal of implant if mobile, entire length and circumference of implant involved.

c. Proper plaque control measures.

\section{E. Improper Angulations (Fig. 4)}

a. Correct any implant malposition at the time of implant placement

b. Removal of implant if necessary (if angulation is not possible to correct)

c. Proper oral hygiene

d. In case of recession or membrane exposure, adequate bands of keratinized tissue can be created by flap positioning or connective tissue grafting at the time of or after implant placement.

\section{F. Implant Complications related to Immediate Implant Placement into Extraction Sites}

a. Correct any implant malposition at the time of implant placement

b. Removal of implant if necessary (if angulation is not possible to correct)

c. Proper oral hygiene

d. In case of recession or membrane exposure, adequate bands of keratinized tissue can be created by flap positioning or connective tissue grafting at the time of or after implant placement. 


\section{G. Complications related to Immediately loaded Dental Implants}

a. If immediately restored implants are found to be mobile within a short time after placement, it may be possible to save them by eliminating or minimizing forces on them.

b. Regular recall appointments.

\section{H. Implant Failures}

a. Diagnose and identify the failed implant.

b. Note the clinical signs: Mobility, edema, pain, pus, bleeding and radiographic signs of peri-implant bone loss.

c. In any case of implant failure where mobility is apparent, the implant should be removed immediately.

d. Replacement of failed implant.

\section{IMPLANT MAINTENANCE}

One of the key factors for long term success of implants is the maintenance of the healthy tissues around it. Implant should have accessible embrasure widths for maintenance with polished collars for prevention of plaque formations. Scaling is to be done delicately to avoid scratches with a plastic scaler. Chlorhexidine gluconate may be used as an irrigant.

Patient must be asked to maintain plaque control. A soft or extrasoft toothbrush must be used. Use of floss and interdental aids may be encouraged.

\section{CONCLUSION}

The ultimate success of implants is not only based on diagnosis, evaluation, treatment planning but also on having a knowledge regarding the complications of implants and their fruitful management. In short it is always better to remember: 'Prevention is better than cure' and 'a stitch in time saves nine.'

\section{REFERENCES}

1. Bjork A. The use of metallic implants in the study of facial growth in children: Method and application. Am I Phys Anthropol 1968;29:243.

2. Proffit WR, Fields HW, Sarver DM. Contemporary orthodontics (4th ed). Mosby, 2007;298.
3. Newman MG, Takei HH, Curranza FA. Advanced implant surgery and bone grafting techniques. In: Clinical periodontology (9th ed). WB Saunders Company 2002;909-21.

4. Esposito M, Hirsch J, Lekholm U, Thomsen P. Differential diagnosis and treatment strategies for biologic complications and failing oral implants: A review of the literature. Int J Oral Maxillofac Implants 1999;14:473-90.

5. Paquette DW, Brodala N, William RC. Risk factors for endosseous dental implant failure. Dent Clin North Am 2006;50:361-74.

6. Goodacre CJ, Kan JYK, Rungcharassaeng K. Clinical complications of osseointegrated implants. J Prosthet Dent 1999;81:537-52.

7. Misch K, Wang HL. Implant surgery complications: Etiology and treatment. Implant Dent 2008;17:159-66.

8. Froum SJ. Dental implant complications: Etiology, prevention, and treatment (1st ed). Blackwell Publishing, 2010:110-18.

9. Albrektsson T, Zarb G, Worthington P, Eriksson A. The longterm efficacy of currently used dental implants: A review and proposed criteria of success. Int J Oral Maxillofac Implants 1986;1:11-25.

10. Roos J, Sennerby L, Leckholm U, Jemt T, Grodahl K, Albrektsson T. A qualitative and quantitative method for evaluating implant success: A 5-year retrospective analysis of the Brånemark implant. Int J Oral Maxillofac Implant 1997;12:504-14.

11. Rosenberg ES, Torosian J. An evaluation of differences and similarities observed in fixture failure of five distinct implant systems. Pract Periodont Aesthet Dent 1998;10:687-98; quiz 700.

12. Balshi TJ. An analysis and management of fractured implants: A clinical report. Int J Oral Maxillofac Implants 1996;11:660-66.

\section{ABOUT THE AUTHORS}

\section{Smita Vaidya (Corresponding Author)}

Associate Professor, Department of Prosthodontics, Government Dental College, Aurangabad, Maharashtra, India, e-mail: smitakhalikar@yahoo.com

\section{Arun Khalikar}

Professor and Head, Department of Prosthodontics, Government Dental College, Nagpur, Maharashtra, India

\section{Shankar P Dange}

Professor and Head, Department of Prosthodontics, Government Dental College, Aurangabad, Maharashtra, India

\section{Rujit Desai}

Postgraduate Student, Department of Prosthodontics, Government Dental College, Aurangabad, Maharashtra, India 